\title{
Teneurs plasmatiques en FSH et LH des agneaux mâles et femelles issus de béliers Lacaune prolifiques et non prolifiques
}

\author{
G. RICORDEAU, M.R. BLANC * et L. BODIN \\ avec la collaboration technique de F. Eychenne, R. Mollaret, \\ J.C. Poirier * et D. Lajous \\ I.N.R.A., Station d'Amélioration génétique des Animaux \\ Centre de Recherches de Toulouse, B.P. 12, Auzeville, F 31320 Castanet-Tolosan \\ * I.N.R.A., Station de Physiologie de la Reproduction \\ Centre de Recherches de Tours, B.P. 1, F 37380 Nouzilly
}

Résumé

Cette étude a pour but de mesurer les teneurs en FSH et LH plasmatique des produits mâles et femelles et la croissance testiculaire des mâles issus de 16 pères Lacaune sélectionnés d'après leur index de prolificité en fermes $(8$ prolifiques $\mathbf{H}$ et 8 non prolifiques B) et de mères Romanov et F1 Lacaune $\times$ Romanov. Elle porte sur 161 agneaux dont 100 mâles et 61 femelles. Les taux de FSH et de LH sont mesurés sur un seul prélèvement de sang à $3,5,7,9$ et 11 semaines; le diamètre testiculaire est mesuré à 7,10 et 14 semaines.

Chez les mâles, le taux de FSH est maximum à 5 semaines $(3,6 \mathrm{ng} / \mathrm{ml})$; il est indépendant du type de naissance, du type génétique des agneaux et de l'index de prolificité de leur père. Intra-type génétique, le diamètre testiculaire est indépendant de l'index du père. Chez les femelles, le maximum de FSH est plus élevé $(8,2 \mathrm{ng})$ et atteint dès 3 semaines. L'effet de l'index du père est très significatif à 3 et 5 semaines et l'effet du type génétique très significatif à 3 semaines, alors que les effets du type de naissance et du poids vif ne sont jamais significatifs. Le taux de FSH à 5 semaines des 58 agnelles croisées est corrélé de façon significative avec leur taux d'ovulation mesuré à 9-10 mois. Ces résultats indiquent que le taux de FSH des agnelles pourrait constituer un critère précoce pour indexer les béliers. En ce qui concerne les concentrations en LH plasmatique des agneaux mâles et femelles, tous les effets (type de naissance, type génétique et index père) sont non significatifs.

Mots clés : Ovins, Lacaune, Romanov, prolificité, taux d'ovulation, taille testiculaire, gonadotropines.

\section{Summary}

Plasma FSH and LH in lambs of Lacaune sires with high and low polificacy indices

The purpose of this study was to examine the plasma FSH and LH levels of male and female lambs and the testicular growth of males issued from 16 Lacaune rams selected 
on their prolificacy index in farms (8 prolific and 8 non prolific rams) and from Romanov and F1 Lacaune $\times$ Romanov ewes. A total of 161 lambs (100 males and 61 females) was observed. Plasma FSH and LH were measured on single blood samples collected at 3, 5, 7, 9 and 11 weeks of age. The testicular diameter was measured at 7, 10 and 14 weeks.

In males, plasma FSH reached a maximum at 5 weeks $(3,6 \mathrm{ng} / \mathrm{ml})$; it was independent of birth type, lamb genotype and sire prolificacy index. Within a genotype, testicular diameter was independent of the sire's index. In females, the maximum concentration of plasma FSH was higher than that of males $(8.2 \mathrm{ng} / \mathrm{ml})$ and was reached as early as 3 weeks of age. The effect of sire prolificacy index was very significant $(P<0.01)$ at 3 and 5 weeks, when effects of birth-type and liveweight are never significant. At 5 weeks of age, FSH plasma concentration of the 58 crossbred ewe lambs was related to their ovulation rate observed at 10 months of age. These results show that plasma FSH levels in ewelambs could constitute an early criterion of ram selection. Plasma LH in males and females was independent of birth type, lamb genotype and sire prolificacy index.

Key words : Sheep, Lacaune breed, Romanov breed, litter-size, ovulation rate, testis size, gonadotropins.

\section{Introduction}

L'utilisation comme critère précoce de la prolificité des brebis, des teneurs plasmatiques en LH (1) des jeunes agneaux, a donné des résultats variables, du fait notamment de la pulsatilité des mesures, des différences dans l'évolution des teneurs avec l'âge, de la mise en œuvre d'effectifs limités présentant une variabilité insuffisante (cf. LAND \& CARR, 1979 ; RiCORDEAU et al., 1979). Les mesures concernant les teneurs plasmatiques en FSH sont plus récentes et n'ont encore fait l'objet que d'observations préliminaires : Foster et al., 1975 ; LeE et al., 1976 ; Findlay \& Bindon, 1976 ; HoCHEREAU de REVIERS et al., 1980 ; Walton et al., 1980; SAVOIE et al., 1981 ; LAFORTUNe et al., 1982 ; SANFORd et al., 1982 ; FitZgerald \& ButLer, 1982. Cependant ,elles sont beaucoup moins variables que les mesures $\mathbf{L H}$, aussi a priori présentent-elles plus d'intérêt.

Pour estimer valablement les relations entre les critères précoces mesurables chez les jeunes et les performances ultérieures de reproduction, nous avons plusieurs approches possibles. Dans cette étude, nous avons retenu la solution expérimentale qui consiste à sélectionner directement des béliers indexés sur la prolificité de leurs filles, et à mesurer la réponse indirecte sur les teneurs en FSH et LH de leurs produits mâles et femelles et la croissance testiculaire des produits mâles, afin de discriminer les critères qui sont en corrélation forte avec la prolificité. Cette solution résulte de la possibilité qui nous était offerte de choisir des béliers ayant des index de prolificité extrêmes, dans le cadre des programmes de testage Lacaune (Lait et viande) réalisés en fermes en utilisant l'insémination artificielle (I.A.).

(1) LH : hormone lutéostimulante (luteinizing hormone).

FSH : hormone folliculostimulante (follicle stimulating hormone). 


\section{Matériel et méthodes}

\section{A. Protocole}

En juillet 1981, parmi les 144 béliers Lacaune indexés (2) disponibles au Centre d'I.A. Ovitest, 16 sont sélectionnés (fig. 1) dont 8 avec un index négatif (béliers $B$ : index moyen $=-0,052$; moyenne brute des filles $=1,18$ ) et 8 avec un index positif (béliers $\mathrm{H}$ : index moyen $=+0,073$; moyenne brute des filles $=1,55$ ). Ces 16 pères servent à inséminer 163 brebis de 2 à 6 ans du troupeau INRA de Toulouse, dont 102 Romanov et 61 F1 Lacaune $\times$ Romanov. Les inséminations sont faites le 16 août 1981, sur œstrus induit (éponges vaginales avec $30 \mathrm{mg}$ de FGA pendant

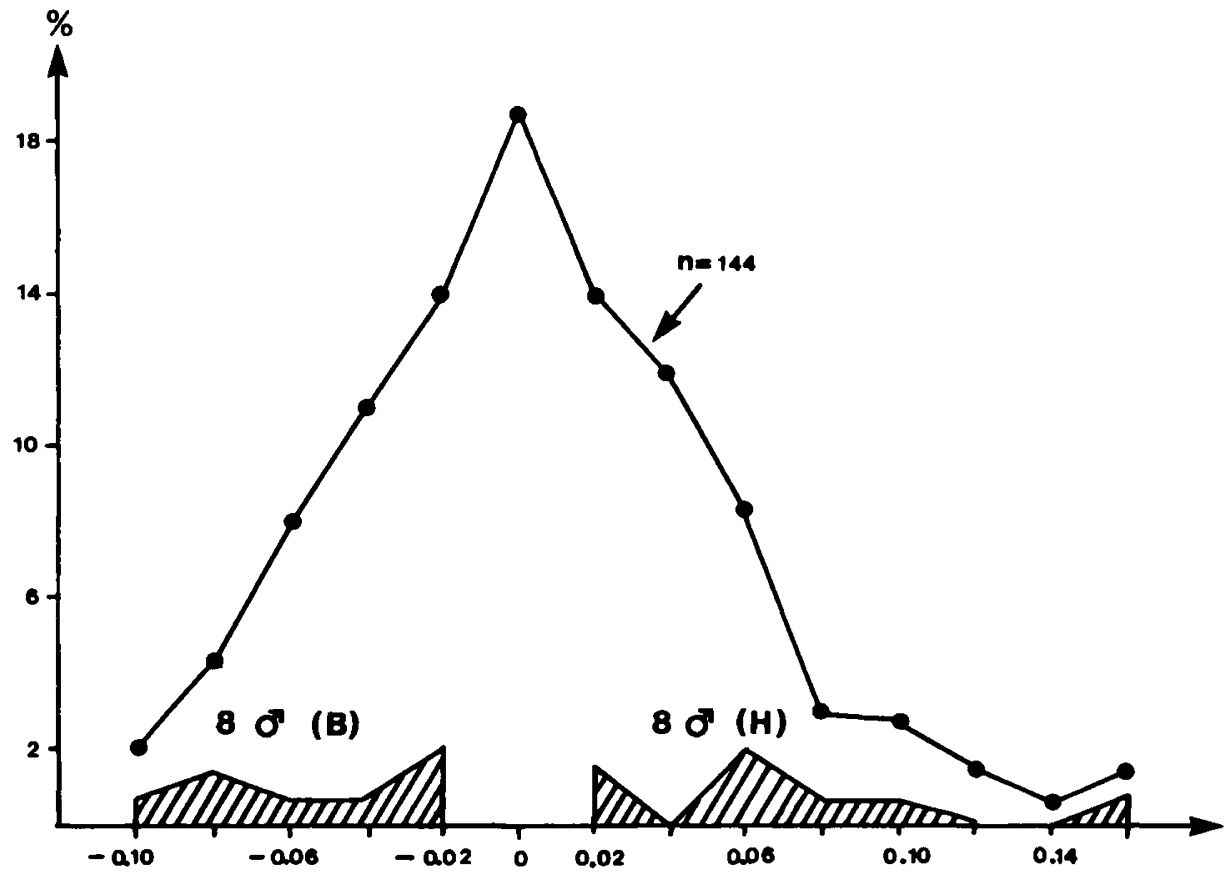

FIG. 1

Index des bolliers

Répartition des 16 béliers prolifiques et non prolifiques parmi les 144 béliers Lacaune indexés sur la prolificité de leurs filles.

Distribution of the 16 prolific and non prolific sires among the 144 Lacaune sires evaluated for litter size of their daughters.

(2) Les index prolificité $\left(I_{i}=C D \times \vec{D}_{i}\right)$ sont calculés par la méthode de comparaison aux contemporaines des mêmes troupeaux (Foulley \& ELSEN, 1977), d'après la prolificité des agnelles à la première mise bas de 14 à 16 mois. $C D$ est le coefficient de détermination dans lequel l'héritabilité de la prolificité est estimée à 0,08 (Bodin et al., 1979) et $\overline{\mathrm{D}}_{\mathrm{i}}$ est l'écart moyen entre la prolificité des filles et celle des contemporaines. 
12 jours et 200 U.I de PMSG à la dépose) avec de la semence fraîche et standardisée. Les agnelages ont lieu en moyenne le 10 janvier 1982. Compte tenu des origines et des types de naissance, 161 agneaux sont retenus, dont $97 \mathrm{~F} 1$ et 64 Backcross (BC) Lacaune, soit 100 mâles et 61 femelles (tabl. 1). Les agneaux sont élevés sous la mère — sauf 10 p. 100 en allaitement artificiel — et tous sevrés à 56 jours.

\section{TABleau 1}

Répartition des 161 agneaux croisés, suivant leur sexe, type de naissance, type génétique et l'index prolificité de leur père.

Distribution of 161 crossbred lambs, by sex, birth-type, genotype and sire prolificacy index.

\begin{tabular}{|c|c|c|c|c|c|c|}
\hline \multirow{2}{*}{ Sexe } & \multirow{2}{*}{$\begin{array}{c}\text { Type } \\
\text { de naissance }\end{array}$} & \multicolumn{2}{|c|}{ BC Lacaune } & \multicolumn{2}{|c|}{$\mathrm{F} 1$} & \multirow{2}{*}{$\mathbf{n}$} \\
\hline & & B & $\mathbf{H}$ & B & $\mathbf{H}$ & \\
\hline Mâles ..... & $\begin{array}{l}1 \text { et } 2 \\
3 \text { et } 4\end{array}$ & $\begin{array}{r}14 \\
5\end{array}$ & $\begin{array}{r}14 \\
4\end{array}$ & $\begin{array}{r}7 \\
23\end{array}$ & $\begin{array}{l}10 \\
23\end{array}$ & 100 \\
\hline Femelles & $\begin{array}{c}2 \\
3 \text { et } 4\end{array}$ & $\begin{array}{l}9 \\
6\end{array}$ & $\begin{array}{l}6 \\
6\end{array}$ & $\begin{array}{l}7 \\
9\end{array}$ & $\begin{array}{r}8 \\
10\end{array}$ & 61 \\
\hline
\end{tabular}

BC Lacaune : 3/4 Lacaune, 1/4 Romanov; F1 : 1/2 Lacaune, 1/2 Romanov.

Parallèlement, des observations sont effectuées sur 20 mâles Romanov nés quelques jours après les agneaux croisés (naissance en moyenne le 16 janvier 1982) mais élevés dans les mêmes conditions. Ces agneaux sont tous allaités sous la mère et sevrés à 56 jours; ils sont issus de 3 pères dont les filles ont un taux moyen d'ovulation de 2,8 à 9 mois.

\section{B. Mesures réalisées}

\section{Prélèvements sanguins}

Pour les agneaux F1 et BC Lacaune, les échantillons de sang sont prélevés à 3, $5,7,9,11$ semaines. Pour les agneaux Romanov, les prélèvements ont lieu à 4, 6, 8 et 10 semaines. A chaque âge, on effectue un seul prélèvement entre $9 \mathrm{~h}$ et $10 \mathrm{~h}$. Le sang est mis dans la glace fondante jusqu'à centrifugation au maximum une heure plus tard. Le plasma est pipeté et conservé à $-12^{\circ} \mathrm{C}$ jusqu'à l'analyse. La concentration en FSH est mesurée par dosage radioimmunologique homologue selon la méthode décrite par Blanc \& Polrier (1979) et exprimée en ng de FSH, HG 225 par $\mathrm{ml}$ de plasma (3). La concentration en $\mathbf{L H}$ plasmatique est mesurée par un dosage radioimmunologique homologue, spécifique et très sensible (PoIRIER \& BLANC, non

(3) Préparation due à l'amabilité du $\mathrm{D}^{\mathrm{r}}$ GRIMeK, Madison, Wisconsin, U.S.A. : $1 \mathrm{mg}$ FSH, HG 225 immunologiquement équivalent à $14 \mathrm{mg}$ de FSH NIH-S3. 
publié) et exprimée en $\mathrm{ng}$ de LH 1051 par $\mathrm{ml}$ de plasma (4). Les déterminations de FSH et de LH sont faites séparément, mais tous les échantillons sont analysés en même temps afin de diminuer la variabilité des résultats.

\section{Mesures testiculaires et croissance pondérale}

Les diamètres testiculaires sont mesurés à 50,71 et 98 jours sur les croisés, à 50 , 71,98 et 120 jours sur les Romanov. Les mesures sont prises au pied à coulisse, par le même opérateur, sur le plus grand diamètre antéropostérieur, épaisseur du scrotum incluse (des observations antérieures en races Romanov et Lacaune ont montré que l'épaisseur du scrotum était peu variable).

Pour tous les agneaux, les pesées ont lieu à la naissance et tous les 21 jours jusqu'à l'abattage, ce qui permet de calculer les poids à $30,60,70$ et 90 jours par interpolation linéaire et les 2 gains moyens quotidien (GMQ) de 0 à 30 et de 70 à 90 jours.

\section{Performances de reproduction des agnelles $F 1$ et $B C$ Lacaune}

Les 61 agnelles croisées dont le niveau de FSH est connu, sont conservées pour la reproduction. Leur activité ovarienne est contrôlée en début de saison, par dosage de la progestérone plasmatique les 17 et 27 août 1982. La pesée avant la saillie a lieu le 19 octobre et le taux d'ovulation est mesuré par laparoscopie à 9-10 mois, du 19 octobre au 6 novembre, 5 à 7 jours après la saillie.

\section{Analyse statistique concernant les agneaux croisés}

\section{Teneurs plasmatiques en FSH et $\mathrm{LH}$}

Une première analyse de variance à effets fixés est effectuée sur les mesures enregistrées à $3,5,7,9$ et 11 semaines, séparément pour les mâles et femelles, en tenant compte des facteurs suivants : type de naissance (1 et 2,3 et 4 ), type génétique des agneaux ( $\mathrm{F} 1$ et $\mathrm{BC}$ Lacaune), index prolificité des pères $(\mathrm{H}$ et $\mathrm{B})$ et des interactions type génétique $X$ index père, type génétique $X$ type de naissance. Dans une seconde analyse, nous éliminons le type de naissance des agneaux afin de prendre en compte leur poids vif à 0,30 et 70 jours comme covariables.

Les mesures de LH ou de FSH (notamment celles des agnelles) ayant une distribution légèrement dissymétrique, nous avons appliqué la transformation $\sqrt{\mathrm{x}}$, mais celle-ci ne modifie pas les résultats des tests. Aussi, par souci de simplification, nous avons négligé les données transformées dans la présentation des résultats.

La répétabilité des mesures chez les mâles et femelles est estimée par le coefficient de corrélation intra-classe (analyse hiérarchique père-produits), après correction des effets dus au type génétique et à l'âge : soit $\varrho=\left(\sigma_{\mathrm{g}}^{2}+\sigma_{\mathrm{a}}^{2}\right) /\left(\sigma_{\mathrm{s}}^{2}+\sigma_{\mathrm{a}}^{2}+\sigma_{\mathrm{m}}^{2}\right)$ avec $\sigma_{\mathrm{s}}^{2}=$ variance entre pères; $\sigma_{\mathrm{a}}^{2}=$ variance entre individus intra père et $\sigma_{\mathrm{m}}^{2}=$ variance

(4) Préparation due à l'amabilité du $\mathrm{D}^{\mathrm{r}}$ Combarnous (C.N.R.S., Nouzilly) et équivalente à 2,7 fois la OLH-NIH-S1. 
entre mesures intra individu. La corrélation phénotypique entre les mesures individuelles de FSH et de LH est déduite de la même analyse, à partir des estimations de covariance.

\section{Croissance pondérale}

L'analyse de variance à effets fixés est effectuée en tenant compte du sexe, du type de naissance, du type génétique des agneaux, et de l'index de prolificité de leur père $(\mathbf{H}$ et $\mathbf{B})$.

\section{Résultats}

\section{A. Fertilité et prolificité des 163 mères accouplées aux 16 pères Lacaune $H$ et $B$}

Sur œstrus induit, nous n'observons aucune différence en ce qui concerne le taux de réussite ou la taille de portée des mères inséminées par les béliers prolifiques $(\mathrm{H})$ et non prolifiques (B) : respectivement 72,3 et 73,0 p. $100 ; 2,48$ et 2,49 .

\section{B. Teneurs plasmatiques en FSH}

1. Mâles et femelles issus de pères Lacaune

Le niveau et la variabilité des teneurs en FSH sont très différents entre mâles et femelles, ce qui nous a conduit à effectuer les analyses par sexe.

\section{a. Mâles croisés}

La teneur est maximale de 5 à 7 semaines : 3,6 $\mathrm{ng} / \mathrm{ml}$. Les effets type naissance et index père des agneaux ne sont jamais significatifs. L'effet type génétique et l'interaction type génétique $X$ index père sont significatifs mais à une seule période (tabl. 2). La prise en compte du poids vif des agneaux ( $2^{\mathrm{e}}$ analyse) ne modifie pas les résultats.

\section{b. Femelles croisées}

La concentration diminue régulièrement de 3 à 9 semaines $(8,2$ à $4,6 \mathrm{ng} / \mathrm{ml}$ : fig. 2). L'effet index père est très significatif à 21 et 35 jours, significatif à 49 jours et non significatif au-delà (tabl. 2), la teneur en FSH étant supérieure pour les agnelles issues de pères prolifiques : 10,1 contre $6,3 \mathrm{ng}$ à 21 jours. L'effet type de naissance n'est jamais significatif et l'effet type génétique est significatif à 21 jours, très significatif à 35 jours et non significatif au-delà, les femelles $F 1$ ayant un niveau supérieur aux femelles BC Lacaune : 9,3 contre 6,7 ng à 21 jours. Les 2 interactions ne sont jamais significatives. melles.

La répétabilité des mesures est de 0.49 pour les mâles et de 0.56 pour les fe- 


\section{TABLEAU 2}

Moyennes ajustées des taux plasmatiques de FSH (en $\mathrm{ng} / \mathrm{ml}$ ) des agneaux croisés, en fonction de leur sexe, type de naissance, type génétique et l'index de leur père ( $1^{\text {re }}$ analyse).

Coefficients de régression avec le poids vif en covariable (2* analyse).

Least-squares means of plasma FSH of crossbred lambs, by sex, birth-type, genotype and sire prolificacy index (1st analysis).

Regression coefficients with the liveweight as covariable (2nd analysis).

$1^{\text {re }}$ analyse

\begin{tabular}{|c|c|c|c|c|c|}
\hline \multirow{2}{*}{ Effets } & \multicolumn{5}{|c|}{ Age en semaines } \\
\hline & $3 \mathrm{~s}$ & $5 \mathrm{~s}$ & $7 \mathrm{~s}$ & $9 \mathrm{~s}$ & $11 \mathrm{~s}$ \\
\hline Mâles $(\mu)$ & 2,9 & 3,6 & 3,6 & 2,7 & 2,8 \\
\hline Type de naissance (TN) & NS & NS & NS & NS & NS \\
\hline Type génétique (TG) $\ldots \ldots$ & NS & NS & NS & $\mathbf{S}$ & NS \\
\hline $\mathbf{F 1} \ldots \ldots \ldots \ldots \ldots \ldots$ & 3,0 & 3,8 & 3,5 & 2,5 & 2,8 \\
\hline BC Lacaune .......... & 2,8 & 3,3 & 3,7 & 3,2 & 2,9 \\
\hline Index père (IP) $\ldots \ldots$ & NS & NS & NS & NS & NS \\
\hline $\mathbf{H} \ldots \ldots \ldots \ldots \ldots$ & 2,9 & 3,7 & 3,7 & 2,7 & 2,6 \\
\hline B $\ldots \ldots \ldots \ldots \ldots \ldots$ & 2,9 & 3,6 & 3,5 & 2.8 & 3,0 \\
\hline \multicolumn{6}{|l|}{ Interactions } \\
\hline TG $\times I P$ & NS & NS & $\mathbf{S}$ & NS & NS \\
\hline TG $\times$ TN $\ldots \ldots \ldots \ldots$ & NS & NS & NS & NS & NS \\
\hline Femelles $(\mu)$ & 8,2 & 6,9 & 5,5 & 4,6 & 5,1 \\
\hline Type de naissance & NS & NS & NS & NS & NS \\
\hline Type génétique .. & $\mathbf{S}$ & TS & NS & NS & NS \\
\hline F1 $\ldots \ldots \ldots$ & 9,3 & 8,3 & 6,1 & 4,9 & 5,4 \\
\hline BC Lacaune .... & 6,7 & 5,1 & 4,6 & 4,2 & 4,7 \\
\hline Index père & TS & TS & $\mathrm{S}$ & $\mathrm{N} S \mathrm{~S}$ & NS \\
\hline $\mathbf{H} \ldots$ & 10,1 & 8,7 & 6,6 & 5,4 & 5,6 \\
\hline B $\ldots$ & 6,3 & 5,2 & 4,3 & 3,8 & 4,6 \\
\hline Interactions & NS & NS & NS & NS & NS \\
\hline
\end{tabular}

\section{$2^{e}$ analyse}

Coefficients de régression des taux de FSH sur le poids vif à 0,30 et 70 jours

\begin{tabular}{|c|c|c|c|c|c|c|}
\hline $\begin{array}{l}\text { Mâles } \\
\qquad \begin{array}{l}\text { Naissance } \ldots \ldots \ldots \ldots \ldots \\
30 \mathrm{j} \ldots \ldots \ldots \ldots \ldots \ldots\end{array} \\
70 \mathrm{j} \ldots \ldots \ldots \ldots \ldots \ldots\end{array}$ & NS & $\begin{array}{l}\text { NS } \\
\text { NS }\end{array}$ & $\begin{array}{r}-0,56 \mathrm{TS} \\
\mathrm{NS}\end{array}$ & $\begin{array}{r}-0,54 \text { TS } \\
-0,18 \text { TS } \\
\text { NS }\end{array}$ & $\begin{array}{r}-0,35 \\
0,05\end{array}$ & $\begin{array}{l}\text { S } \\
\text { NS } \\
\text { TS }\end{array}$ \\
\hline Femelles 0,30 et $70 j$ & NS & NS & NS & NS & & NS \\
\hline
\end{tabular}




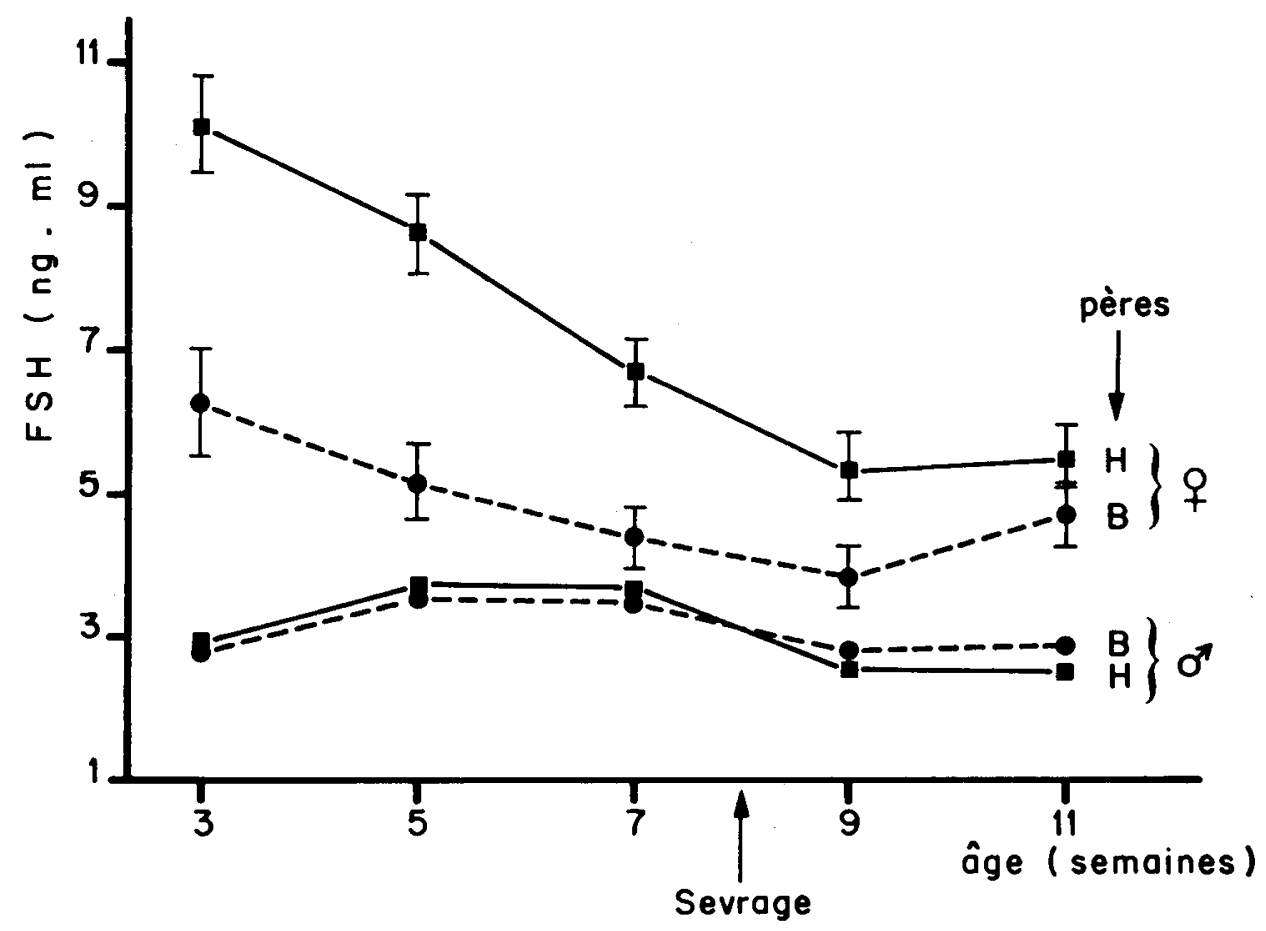

FIG. 2

Evolution de la concentration plasmatique en FSH des agneaux croisés mâles et femelles, nés de pères prolifiques $(H)$ et non prolifiques $(B)$ : moyenne \pm écart type de la moyenne pour les femelles.

Evolution of plasma FSH of male and female crossbred lambs born from prolific $(H)$ and non prolific $(B)$ sires : mean \pm standard error of the mean of females.

\section{Tableau 3}

Mâles Romanov.

Taux de FSH de 4 à 10 semaines et croissance testiculaire de 50 à 120 jours.

Romanov males.

Plasma FSH from 4 to 10 weeks and testis diameter from 50 to 120 days.

\begin{tabular}{c|c|c|c}
\hline \hline \multicolumn{2}{c|}{ Mesures FSH } & \multicolumn{2}{|c}{ Diamètre testiculaire } \\
\cline { 2 - 4 } Age (semaines) & $\mathrm{ng} / \mathrm{ml}$ & Age (jours) & $\mathrm{cm}$ \\
\hline 4 & 5,0 & 50 & 2,15 \\
6 & 5,1 & 71 & 2,97 \\
8 & 4,1 & 98 & 4,27 \\
10 & 3,3 & 120 & 5,23 \\
\hline
\end{tabular}




\section{Mâles Romanov}

La teneur maximale en FSH se situe à 6 semaines, soit au même âge que chez les croisés, mais à un niveau significativement plus élevé : 5,0 et $5,1 \mathrm{ng} / \mathrm{ml}$ pour les Romanov, contre 3,6 pour les croisés (tabl. 3). La répétabilité des mesures est de 0.52 .

\section{Teneurs plasmatiques en $L H$}

Pour les mâles, la teneur est maximale à 9 semaines, alors qu'elle diminue régulièrement de 3 à 9 semaines pour les femelles (tabl. 4). Intra-sexe, les effets type de naissance, type génétique et index père sont tous non significatifs. La répétabilité des mesures est de 0.15 pour les mâles et de 0.21 pour les femelles. Les corrélations entre les taux individuels de $\mathbf{L H}$ et FSH sont respectivement de 0.25 et 0.45 pour les mâles et femelles.

\section{TABLEAU 4}

Moyennes ajustées des taux plasmatiques de $\mathrm{LH}$ (en $\mathrm{ng} / \mathrm{ml}$ ) des agneaux croisés, en fonction de leur sexe, type de naissance, type génétique et de l'index de leur père.

Least-squares means of plasma LH of crossbred lambs by sex, birth-type, genotype and sire prolificacy index.

\begin{tabular}{|c|c|c|c|c|c|}
\hline \multirow{2}{*}{ Effets } & \multicolumn{5}{|c|}{ Age en semaines } \\
\hline & $3 \mathrm{~s}$ & $5 \mathrm{~s}$ & $7 \mathrm{~s}$ & $9 \mathrm{~s}$ & $11 \mathrm{~s}$ \\
\hline Mâles $(\mu)$ & 1,30 & 2,05 & 2,14 & 2,23 & 1,82 \\
\hline Type de naissance et type génétique .. & NS & NS & NS & NS & NS \\
\hline $\begin{array}{r}\text { Index père } \ldots \ldots \ldots \ldots \ldots \ldots \ldots \ldots \\
\mathbf{H} \\
\mathbf{B} \\
\quad \ldots \ldots \ldots \ldots \ldots\end{array}$ & $\begin{array}{l}\text { NS } \\
1,24 \\
1,36\end{array}$ & $\begin{array}{l}\text { NS } \\
2,09 \\
2,02\end{array}$ & $\begin{array}{l}\text { NS } \\
2,15 \\
2,13\end{array}$ & $\begin{array}{l}\text { NS } \\
2,21 \\
2,24\end{array}$ & $\begin{array}{l}\text { NS } \\
1,80 \\
1,83\end{array}$ \\
\hline Femelles $(\mu)$ & 2,14 & 1,95 & 1,52 & 1,26 & 1,48 \\
\hline Type de naissance et type génétique .. & NS & NS & NS & NS & NS \\
\hline 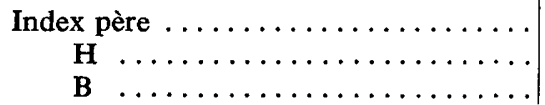 & $\begin{array}{l}\text { NS } \\
2,15 \\
2,13\end{array}$ & $\begin{array}{l}\text { NS } \\
1,92 \\
1,97\end{array}$ & $\begin{array}{l}\mathrm{NS} \\
1,80 \\
1,26\end{array}$ & $\begin{array}{l}\text { NS } \\
1,24 \\
1,29\end{array}$ & $\begin{array}{l}\text { NS } \\
1,42 \\
1,73\end{array}$ \\
\hline
\end{tabular}

\section{Poids vif des agneaux. Relations avec les teneurs en FSH}

Les effets sexe et type de naissance sont très significatifs sur les poids à 30,60 et 90 jours (tabl. 5). L'effet type génétique est significatif sur le poids à 30 jours et très significatif sur le gain moyen de 0 à 30 jours; l'effet index père est également significatif sur ces 2 variables, les agneaux issus de pères prolifiques $\mathbf{H}$ ayant une croissance supérieure à ceux de pères $B$ pendant le premier mois d'allaitement. 


\section{TABLEAU 5}

Moyennes ajustées du poids vif des agneaux croisés suivant leur sexe, type de naissance, type génétique et l'index de leur père. Croissance des mâles Romanov.

Least-squares means of liveweights of crossbreds lambs, by sex, birth-type, genotype and sire prolificacy index. Growth of Romanov males.

\begin{tabular}{|c|c|c|c|c|c|c|}
\hline \multirow{2}{*}{ Effets } & \multicolumn{4}{|c|}{ Poids en kg } & \multicolumn{2}{|c|}{ G.M.Q. en $\mathrm{g}$} \\
\hline & Naissance & $30 \mathrm{j}$ & $60 \mathrm{j}$ & $90 \mathrm{j}$ & $0-30 \mathrm{j}$ & $70-90 \mathrm{j}$ \\
\hline Croisés $(\mu)$ & 3,0 & 9,4 & 18,0 & 28,1 & 216 & 338 \\
\hline $\begin{array}{l}\text { Sexe } \ldots \ldots \ldots \ldots \ldots \\
\text { Mâle } \ldots \ldots \ldots \ldots \\
\text { Femelle } \quad \ldots \ldots \ldots\end{array}$ & $\begin{array}{l}\text { NS } \\
3,0 \\
2,9\end{array}$ & $\begin{array}{l}\text { TS } \\
9,7 \\
9,0\end{array}$ & $\begin{array}{c}\text { TS } \\
18,7 \\
16,8\end{array}$ & $\begin{array}{c}\text { TS } \\
29,8 \\
25,5\end{array}$ & $\begin{array}{c}\text { TS } \\
223 \\
203\end{array}$ & $\begin{array}{l}\text { TS } \\
368 \\
290\end{array}$ \\
\hline $\begin{array}{r}\text { Type de naissance }(\mathrm{TN}) \\
1 \text { et } 2 \ldots \ldots \ldots \ldots \ldots \\
3 \text { et } 4 \ldots \ldots \ldots\end{array}$ & $\begin{array}{l}\text { TS } \\
3,3 \\
2,7\end{array}$ & $\begin{array}{l}\text { TS } \\
9,9 \\
9,0\end{array}$ & $\begin{array}{c}\text { TS } \\
18,9 \\
17,2\end{array}$ & $\begin{array}{c}\text { TS } \\
29,3 \\
27,1\end{array}$ & $\begin{array}{l}\text { NS } \\
221 \\
210\end{array}$ & $\begin{array}{l}\text { NS } \\
344 \\
333\end{array}$ \\
\hline $\begin{array}{l}\text { Type génétique (TG) } \ldots \ldots \\
\text { F1 } \ldots \ldots \ldots \ldots \ldots \\
\text { BC Lacaune } \ldots \ldots \ldots\end{array}$ & $\begin{array}{l}\text { NS } \\
3,0 \\
2,9\end{array}$ & $\begin{array}{c}\mathrm{S} \\
9,2 \\
9,8\end{array}$ & $\begin{array}{c}\text { NS } \\
17,6 \\
18,6\end{array}$ & $\begin{array}{l}\text { NS } \\
27,7 \\
28,8\end{array}$ & $\begin{array}{l}\text { TS } \\
207 \\
229\end{array}$ & $\begin{array}{l}\text { NS } \\
335 \\
343\end{array}$ \\
\hline $\begin{array}{r}\text { Index père (IP) } \ldots \ldots \ldots \\
\mathbf{H} \quad \ldots \ldots \ldots \ldots \ldots \\
\mathbf{B} \quad \ldots \ldots \ldots \ldots\end{array}$ & $\begin{array}{l}\text { NS } \\
3,0 \\
2,9\end{array}$ & $\begin{array}{c}\text { S } \\
9,7 \\
9,1\end{array}$ & $\begin{array}{c}\text { NS } \\
18,3 \\
17,7\end{array}$ & $\begin{array}{c}\text { NS } \\
28,5 \\
27,8\end{array}$ & $\begin{array}{c}S \\
224 \\
207\end{array}$ & $\begin{array}{l}\text { NS } \\
338 \\
338\end{array}$ \\
\hline $\begin{array}{r}3 \text { interactions : sexe } \times \mathrm{TN}, \\
\text { sexe } \times \mathrm{TG}, \mathrm{TN} \times \mathrm{TG} . \\
\end{array}$ & NS & NS & NS & NS & NS & NS \\
\hline Mâles Romanov $(\mu) \ldots .$. & 2,4 & 7,6 & 14,9 & 24,0 & 171 & 306 \\
\hline
\end{tabular}

Chez les agnelles, les taux de FSH sont tous indépendants des poids vifs. Chez les mâles, les taux de FSH de 7 à 11 semaines varient de façon significative, notamment avec le poids de naissance, les coefficients de régression $\mathrm{FSH} /$ poids de naissance étant tous négatifs : tabl. $2,2^{\mathrm{e}}$ analyse.

\section{TABleau 6}

Diamètre testiculaire $(e n \mathrm{~cm})$ des mâles croisés, suivant leur âge, leur type génétique et l'index de leur père.

Testis diameter of crossbred males, by age, genotype and sire prolificacy index.

\begin{tabular}{|c|c|c|c|c|}
\hline \multirow{2}{*}{$\underset{\text { (jours) }}{\text { Age }}$} & \multicolumn{2}{|c|}{ BC Lacaune } & \multicolumn{2}{|c|}{ F1 } \\
\hline & $\mathrm{B}$ & $\mathbf{H}$ & B & $\mathbf{H}$ \\
\hline $\begin{array}{ll}50 & \ldots \\
71 & \ldots \\
98 & \ldots\end{array}$ & $\begin{array}{l}2,15 \\
3,05 \\
4,37\end{array}$ & $\begin{array}{l}2,17 \\
3,08 \\
4,36\end{array}$ & $\begin{array}{l}2,13 \\
3,03 \\
4,21\end{array}$ & $\begin{array}{l}2,16 \\
3,06 \\
4,32\end{array}$ \\
\hline$n \ldots \ldots$ & 19 & 18 & 29 & 30 \\
\hline
\end{tabular}




\section{E. Croissance testiculaire et teneurs en FSH}

En ce qui concerne le diamètre testiculaire, il n'existe aucune différence significative entre types génétiques d'agneaux et intra-type, entre agneaux issus de pères indexés $\mathrm{H}$ et $\mathrm{B}$ (tabl. 6). Seul le taux de FSH à 3 semaines est en corrélation très significative $(0.23$ à 0.30$)$ avec le diamètre testiculaire corrigé - pour le poids vif -de 50 à 98 jours.

Au même âge, le diamètre testiculaire des agneaux Romanov est peu différent de celui des croisés (tabl. 3 et 6 ), mais ces derniers ont des poids nettement supérieurs $(+5,8 \mathrm{~kg}$ à $90 \mathrm{j}:$ tabl. 5).

\section{F. Performances de reproduction des agnelles et teneurs en FSH (tabl. 7)}

Les agnelles issues de pères prolifiques ont un taux d'activité ovarienne à 7-8 mois et un taux d'ovulation à 9-10 mois supérieurs à ceux des agnelles issues de pères non prolifiques : respectivement 64,3 contre 45,4 p. $100 ; 2,35$ contre 1,67 . En ce qui concerne les relations entre index prolificité du père et les critères mesurés sur les filles (taux de FSH de 3 à 7 semaines et taux d'ovulation) on vérifie que les corrélations sont significatives ou très significatives $(+0.36$ et 0.49$)$. La corrélation entre les mesures FSH des agnelles et leur taux d'ovulation à 7-8 mois n'est significativement positive qu'avec la concentration en FSH à 5 semaines.

\section{TABLEAU 7}

Performances de reproduction des agnelles croisées nées de pères prolifiques $(H)$ et non prolifiques $(B)$.

Reproduction performance of crossbred ewe-lambs born from prolific and non prolific sires.

\begin{tabular}{|c|c|c|}
\hline Mesures des agnelles & B & $\mathrm{H}$ \\
\hline Activité ovarienne à $7-8$ mois (p. 100) (a) & $45,4 \quad(33)$ & $64,3 \quad(28)$ \\
\hline Taux d'ovulation à $9-10$ mois $\ldots \ldots \ldots \ldots \ldots$ & $1,67(33)$ & $2,35(26)$ \\
\hline Taille de portée à la première mise bas $\ldots . .$. & $1,7 \quad(29)$ & $2,4 \quad(27)$ \\
\hline
\end{tabular}

(a) Activité ovarienne : pourcentage d'agnelles cyclées estimé à partir des taux de progestérone plasmatique mesurés à 10 jours d'intervalle.

Corrélations moyennes pour les agnelles F1 et BC Lacaune $(\mathrm{n}=58)^{(b)}$

\begin{tabular}{|c|c|c|c|c|c|c|}
\hline Variables & $\begin{array}{l}\text { FSH } \\
3 \mathrm{~s}\end{array}$ & $\begin{array}{l}\text { FSH } \\
5 \mathrm{~s}\end{array}$ & $\begin{array}{l}\text { FSHH } \\
7 \mathrm{~s}\end{array}$ & $\begin{array}{l}\text { FSH } \\
3 \text { à } 7 \mathrm{~s}\end{array}$ & $\begin{array}{l}\text { Poids } \\
\text { à } \\
9 \text { mois }\end{array}$ & $\begin{array}{c}\text { Index } \\
\text { père }\end{array}$ \\
\hline FSH $5 \mathrm{~s}, \ldots \ldots \ldots \ldots \ldots$ & $0,45^{* *}$ & & & & & \\
\hline FSH $7 \mathrm{~s} \ldots \ldots \ldots \ldots \ldots$ & $0,57 * *$ & $0,43 * *$ & & & & \\
\hline FSH 3 à $7 \mathrm{~s} \ldots$ & $0,89 * *$ & $0,75^{* * *}$ & $0,79 * *$ & & & \\
\hline Poids à 9 mois $\ldots \ldots \ldots$ & 0,23 & 0,30 & 0,24 & 0,30 & & \\
\hline Index prolificité du père.. & 0,24 & $0,39 * *$ & $0,32 *$ & $0,36 *$ & 0,24 & \\
\hline Taux d'ovulation ........ & 0,14 & $0,34^{*}$ & 0,24 & 0,28 & $0,40 * *$ & $0,49 * *$ \\
\hline
\end{tabular}

(b) Corrélations moyennes calculées après transformation $\mathrm{Z}$ des corrélations intra type génétique. 


\section{Discussion}

\section{A. Taux plasmatique de FSH}

Cette étude fait apparaître des différences essentielles entre mâles et femelles dans l'évolution, le niveau et la variabilité du taux de FSH.

Chez les mâles, le maximum est obtenu vers 6 semaines et les différences entre «index père » ne sont pas significatives. Les teneurs observées sur les Romanov sont supérieures à celles des croisés Romanov $\times$ Lacaune, mais ce résultat doit être vérifié sur un échantillon plus important.

Chez les femelles, les écarts entre «index père " sont très significatifs dès la première mesure à 21 jours, ce qui signifie bien que la sélection directe des béliers sur leur index prolificité a augmenté indirectement le taux de FSH de leurs filles. C'est là une réponse indiscutable puisque les filles issues des béliers prolifiques et non prolifiques ont des taux d'ovulation et des tailles de portée très différents. Ce résultat est à rapprocher des mesures effectuées par MAUleON \& THIMONIER en 1971 (données non publiées) sur des agnelles de 5 types génétiques abattues moins de 5 jours après la naissance, mesures qui traduisent une augmentation du contenu hypophysaire en FSH en fonction du pourcentage de sang Romanov (tabl. 8). Il confirme également les observations obtenues par FindLaY \& Bindon (1976) à 1 mois, sur 26 agnelles Mérinos appartenant aux 4 troupeaux de l'expérimentation du C.S.I.R.O. : taux de FSH significativement supérieur dans les lots $T$ (sélectionné pour la prolificité) et $B$ (Booroola) par rapport aux lots $\mathrm{C}$ (témoin) et $\mathrm{O}$ (sélectionné contre la prolificité). Ce sont là, à notre connaissance, les seuls travaux antérieurs suggérant une relation FSH-prolificité sur de jeunes agnelles.

\section{TABleau 8}

Teneur et contenu hypophysaire en FSH des agnelles Berrichonnes, Romanov et croisées, à la naissance

(d'après Mauleon \& Thimonier, 1971, non publié).

Concentration and quantity of FSH hypophysis of Berrichon, Romanov and crossbred ewe-lambs at birth.

\begin{tabular}{|c|c|c|c|}
\hline $\begin{array}{l}\text { Type génétique } \\
\text { des agnelles }\end{array}$ & $\begin{array}{c}\text { Poids moyen } \\
\text { d'une hypophyse } \\
\text { seche }\end{array}$ & $\begin{array}{l}\text { Concentration } \\
\text { en FSH (1) }\end{array}$ & $\begin{array}{l}\text { Contenu en FSH } \\
\text { d'une hypophyse } \\
\text { sèche }\end{array}$ \\
\hline Berrichon du Cher.. & 22.6 & 1,0 & 22,6 \\
\hline $\begin{array}{l}1 / 4 \mathrm{ROM}, 3 / 4 \mathrm{BC} \ldots \\
\mathrm{F} 1 \ldots \ldots \ldots \ldots\end{array}$ & $\begin{array}{l}18,9 \\
20,9\end{array}$ & $\begin{array}{l}1,8 \\
1,8\end{array}$ & $\begin{array}{l}34,0 \\
37,6\end{array}$ \\
\hline 3/4 ROM, 1/4 BC ... & 21,9 & 2,5 & 54,7 \\
\hline Romanov ....... & 14,4 & 6,0 & 86,4 \\
\hline
\end{tabular}

5 agnelles par type génétique abattues de 0 à 4 jours, en janvier 1971.

(1) $\mu \mathrm{g}$ d'équivalent FSH, NIH par $\mathrm{mg}$ de poudre acétonique. 
L'évolution des teneurs observées chez les agnelles dans notre expérimentation est sensiblement différente de celles enregistrées par Foster et al. (1975), Fitzgerald \& ButLer (1982), qui n'utilisent pas tout à fait la même technique de dosage et qui travaillent sur un petit nombre d'animaux. Pour éprouver nos résultats, nous avons effectué des mesures complémentaires sur 200 agnelles Lacaune, Romanov, Ile de France et croisés Romanov $\times$ Ile de France : pour tous ces types génétiques nous constatons que l'évolution du taux de FSH est tout à fait comparable à celle observée dans la présente étude.

\section{B. Croissance testiculaire}

L'absence de différence entre les jeunes mâles issus de pères Lacaune prolifiques et non prolifiques prouve que, dans notre étude, la croissance testiculaire avant la puberté est un critère peu en relation avec les caractères de reproduction des femelles. On note cependant que le diamètre testiculaire de 50 à 100 jours est en corrélation significative avec le taux de FSH à 21 jours, mais uniquement à cet âge, alors qu'en race Romanov toutes les corrélations entre mesures testiculaires et taux de $\mathbf{L H}$ de 28 à 56 jours étaient non significatives (RICORDEAU et al., 1979).

\section{Taux plasmatique de $L H$}

On sait que la sécrétion de LH chez le jeune agneau mâle ou femelle possède un caractère pulsatile, ce qui rend difficile l'interprétation de nos mesures de LH comme critère précoce, puisque nous n'avons effectué qu'une seule mesure à chaque âge. Cependant, on peut supposer que ce critère a peu d'intérêt puisque nous n'observons aucun effet significatif de l'index prolificité des pères sur le taux de LH de leurs produits mâles et femelles. Ce résultat va dans le même sens que ceux obtenus par ailleurs si l'on se limite aux seules analyses intra-race : indépendance entre le taux de LH de 4 à 8 semaines ( 3 mesures à chaque âge) de mâles Romanov et le taux d'ovulation de leurs demi-sœurs de père (RICORDEAU et al., 1979); indépendance entre le taux de LH à 4 semaines (1 mesure ou une série de mesures) des femelles Mérinos et leur prolificité aux 2 premiers agnelages (BINDON \& PIPER, 1976), ou entre le taux de $\mathbf{L H}$ à partir de 9 semaines et le taux d'ovulation des agnelles de 4 types génétiques (ECHTERNKAMP \& LASTER, 1976).

\section{Conclusion}

Ces observations préliminaires semblent indiquer que le taux plasmatique de FSH des agnelles peut constituer un critère précoce pour l'indexation des béliers. Le critère FSH est d'autant plus intéressant qu'il est indépendant du type de naissance ou du poids des agneaux et qu'il peut se mesurer sur toutes les agnelles nées vivantes. Cependant, ces données ont été obtenues avec des agnelles croisées et sur un effectif limité. Il importe donc d'entreprendre des études complémentaires en race pure, pour estimer les paramètres génétiques (héritabilité du taux de $\mathrm{FSH}$, corrélation génétique avec les performances initiales et futures de reproduction) et mesurer de façon plus précise l'intérêt de ce critère pour la sélection. 


\section{Remerciements}

Cette étude a été réalisée dans le cadre de l'ATP «Productivité Numérique » financée par l'I.N.R.A. Les auteurs tiennent à remercier B. BiBe et J. Pelletier qui ont facilité la mise en place de cette expérimentation et les responsables du Centre d'I.A. Ovitest qui ont permis la réalisation du protocole concernant l'utilisation des béliers Lacaune des programmes de testage lait et viande.

\section{Références bibliographiques}

BlanC M.R., Poirier J.C., 1979. A new homologous radioimmunoassay for ovine follicle stimulating hormone : development and characterization. Ann. Biol. Anim. Biochim. Biophys., 19, 1011-1026.

Bindon B.M., Piper L.R., 1976. Assessement of new and traditional techniques of selection for reproduction rate. In : TOMES G.J., ROBERTSON D.E., LightFOOD R.J. (ed.), Sheep Breeding, 2nd Ed., 387-401, Butterworths, London.

Bodin L., Ricordeau G., Tchamitchian L., Poujardieu B., 1979. Paramètres génétiques de la prolificité naturelle et induite des agnelles Lacaune. $5^{\text {ss }}$ Journées de la Recherche Ovine et Caprine, I.N.R.A.-I.T.O.V.I.C., Paris, 2-3 décembre 1979, l'Amélioration génétique des espèces ovine et caprine, 224-238.

EChternKamp S.E., LASTer D.B., 1976. Plasma LH concentrations for prepubertal, postpubertal, anestrous and cyclic ewes of varying fecundity. J. Anim. Sci., 42, 444-447.

Findlay J.K., Bindon B.M., 1976. Plasma FSH in Merino lambs selected for fecundity. J. Reprod. Fert., 46, 515-516.

Fitzgerald J., Butler W.R., 1982. Seasonal effects and hormonal patterns related to puberty in ewe lambs. Biol. Reprod., 27, 853-863.

FosTer D.L., J JFFE R.B., Niswender G.D., 1975. Sequential patterns of circulating LH and FSH in female sheep during the early postnatal period : effect of gonadectomy. Endocrinology, 96, 15-22.

Foster D.L., Lemons J.A., JAFFe R.B., Niswender G.D., 1975. Sequential patterns of circulating luteinizing hormone and Follicle-stimulating hormone in female sheep from early postnatal life through the first estrous cycles. Endocrinology, 97, 985-993.

Foulley J.L., ELSEN J.M., 1977. Relations a priori entre BLUP, méthode de comparaison aux contemporaines et méthodes des différences cumulées en vue de l'évaluation des pères. Ann. Génét. Sél. Anim., 9, 307-326.

Hochereau de Reviers M.T., Blanc M.R., Courot M., Garnier D.H., Pelletier J., PoIRIER J.C., 1980. Hormonal profiles and testicular parameters in the lamb. In : Testicular Development, Structure, and Function, 237-247, Raven Press, New York.

Lafortune E., Hochereau de Reviers M.T., Perreau C., Blanc M.R., Orgeur P., PelleTIER J., Terqui M., 1982. Evolutions comparées des teneurs plasmatiques de FSH, LH et la testosterone entre la naissance et la puberté chez l'agneau en fonction de la saison et de la race. Ann. Endocrinol., 43, 124 (Abstr.).

LAND R.B., CARR W.R., 1979. Reproduction in Domestic mammals. In : Genetic variation in Hormone systems, vol. 1, 89-112, Shire, J.G.M. Ed., C.R.C. Press, Boca Raton, Florida, U.S.A.

Lee V.W.K., Cumming I.A., de Kretser D.M., Findlay J.K., Hudson B., KeOgh E.J., 1976. Regulation of gonadotrophin secretion in rams from birth to sexual maturity. I. Plasma LH, FSH and testosterone levels. J. Reprod. Fert., 46, 1-6.

Ricordeau G., Pelletier J., Courot M., Thimonier J., 1979. Phenotypic and genetic relationships between endocrine criteria and testicular measurements of young Romanov rams and the ovulation rates at 8 months of their half-sisters. Ann. Génét. Sél. Anim., 11, 145-159. 
Sanford L.M., Palmer W.M., Howland B.E., 1982. Influence of age and breed on circulating LH, FSH and testosterone levels in the ram. Can. J. Anim. Sci., 62, 767-776.

Savoie S., Bourel B., Hamel R., Buithieu M., Jequier J.C., Bertrand J., Saez J.M., COLLU R., DuchaRME J.R., 1981. Circulating LH, FSH, prolactin, testosterone, $\Delta_{4}$-androstenedione, dehydroepiandrosterone sulfate and cortisol levels in the fetus in late gestation and in newborn male and female lambs. Hormone Res., 15, 122-131.

Walton J.S., Evins J.D., Hillard M.A., Waites G.M.H., 1980. Follicle-stimulating hormone release in hemicastrated prepubertal rams and its relationship to testicular development. J. Endocrinol., 80, 141-152. 